\title{
SPIN DENSITY IN REAL AND MOMENTUM SPACE IN MULTI-ATOM ALLOYS BY KKR-CPA METHOD
}

\author{
S. KAPRZYK \\ Faculty of Physics and Nuclear Techniques, Academy of Mining and Metallurgy \\ Al. Mickiewicza 30, 30-073 Kraków, Poland
}

The application of the spin-polarized version of multiple scattering theory for obtaining electron charge and spin densities in both real and momentum spaces of concentrated, multi-atom disordered alloys is presented. This method is based on the Korringa-Kohn-Rostoker (KKR) band structure approach and coherent potential approximation (CPA) method. The effective one-electron potential is constructed within local spin density approximation. The magnetic neutron form factors are in real space of our main interest. With the recent developments of new synchrotron photon sources, the Compton profile becomes the most interesting target in momentum space. In the most of examples, spin momentum density and its specific structure due to Fermi surface will be shown. To get accurate enough description in momentum space and quantity like Compton profile, the determination of the Fermi surface must be done with high precision. In this context we show how to apply generalized Lloyd formula for accurate determination of the Fermi level. Also we show how to use efficiently complex energy integration method for the computation of matrix elements, $G(r, r)$ or $G(p, p)$, of the KKR-CPA Green function. Results for the iron-silicon ferromagnetic binary alloys and half-metallic ferromagnetic Heuslex alloys are presented.

PACS numbers: $71.15 . \mathrm{Cr}, 71.18 .+\mathrm{y}, 78.90 .+\mathrm{t}$

\section{Introduction}

The aim of this article is to show how using the KKR-CPA theory we may get electron spin density both in real (or, configuration) and momentum space. We will refer to them frequently also as electron spin density and spin momentum density, respectively. This approach was already proved very successful in study of many physical properties such as band spectroscopy, phase stabilities, transport and variety of magnetic related issues. The most spectacular application of KKR-CPA formalism is a first-principles theory of ferromagnetic phase transitions in metals by Gyorffy et al. [1]. For a set of review articles with the references cited, the interested reader may look in Ref. [2]. As such calculations are in fact based on the first-principles concepts and does not involve any adjustable parameters, they become very attractive. To make them feasible and applicable for real system, we 
- use extensively the advantages offered by analycity of the Green function (GF) (referred frequently as Herglotz properties) proved in KKR-CPA theory [3]. The analycity of GF is a very crucial point, and in fact keeping the full form of GF both in real and momentum spaces becomes very critical, as dropping off any part of GF may introduce spurious singularities on complex energy plane. This will immediately prevent the applying of the complex energy contour integration technique. The way the self-consistent procedure is implemented in our KKR-CPA computer code is similar to that of Moruzzi et al. [4] developed for transition metal only. In the first step, when preparing the input data, we assume that we have already the atomic electronic charges, or effective potentials in the form appropriate for the random muffin-tin model. In the second step, we build up the GF in the real space. Then we carry out integration on energy axis up to the Fermi energy $E_{\mathrm{F}}$, in order to find electron densities. This integration is done on the complex energy contour with end point $E_{\mathrm{F}}$, found earlier from generalized Lloyd formula [5]. The self-consistency pu-scedure is set up within the framework of the density functional theory using local spin density (LSD) approximation. In the last stage of computation when all electron spin densities become converged and stable the relevant physical quantities are computed. As most of them are done with the standard method, we will pay only a special attention to problems, which are not so obvious such as treating accurately breaks in $3 d$-momentum space due to Fermi surface and its identification in the structure of Compton profiles.

\section{General formulation}

The presentation in this section is intended to delineate the connection between momentum density and the ground-state energy. The essence of such approach is based on the method proposed by Lam and Platzman [6]. The method uses density functional techniques of Ilohenberg-Kohn (HK) of the inhomogeneous electron gas. Generally, we are looking for the ground state solution of the Schrödinger equation of the solid with the IIamiltonian $H$ including all the many particle interactions $\left(\hbar=e^{2} / 2=2 m=1\right)$ :

$$
H=T+U_{\mathrm{c}}+U_{\mathrm{e}-\mathrm{n}},
$$

with

$$
\begin{aligned}
& T=\sum_{s=(+,-)} \int \mathrm{d} \boldsymbol{r} \nabla \Psi^{+}(s, r) \nabla \Psi(s, \boldsymbol{r}), \\
& U_{\mathrm{c}}=\frac{1}{2} \sum_{s^{\prime}, s=(+,-)} \int \mathrm{d} \boldsymbol{r}^{\prime} \int \mathrm{d} \boldsymbol{r} \Psi^{+}\left(s^{\prime}, \boldsymbol{r}^{\prime}\right) \Psi^{+}(s, \boldsymbol{r}) \frac{e^{2}}{\left|\boldsymbol{r}^{\prime}-\boldsymbol{r}\right|} \Psi(s, \boldsymbol{r}) \Psi\left(s^{\prime}, \boldsymbol{r}^{\prime}\right), \\
& U_{\mathrm{e}-\mathrm{n}}=\sum_{s=(+,-)} \int \mathrm{d} \boldsymbol{r} \Psi^{+}(s, \boldsymbol{r}) \sum_{n} \frac{-Z_{n} e^{2}}{\left|\boldsymbol{r}-\boldsymbol{R}_{n}\right|} \Psi(s, \boldsymbol{r}) .
\end{aligned}
$$

In Eq. (2.1) $T$ is the operator of the electron kinetic energy, $U_{\mathrm{c}}$ is the Coulomb interaction between electrons, and $U_{\mathrm{e}-\mathrm{n}}$ is the Coulomb interaction between electrons and the atomic nucleus. These operators in (2.1) are written in terms of conjugate second-quantized field operators $\Psi(s, r)$ and $\Psi^{+}(s, r)$. We assume that 
nucleus with the atomic order number $Z_{n}$ is frozen in the position indicated by vector $\boldsymbol{R}_{n}$. The electron spin density matrix for such system in the ground state $|0\rangle$ is then defined as

$$
\rho_{s s^{\prime}}(r)=\left\langle 0\left|\Psi^{+}\left(s^{\prime}, r\right) \Psi(s, r)\right| 0\right\rangle .
$$

It is convenient to write $\rho(r)$ defined in (2.3) also in the following form:

$$
\rho(r)=\frac{1}{2}[n(r) \mathbf{1}+\boldsymbol{m}(\boldsymbol{r}) \cdot \sigma],
$$

where $n(r)$ is the charge density function, $m(r)$ is the spin density vector function and $\sigma=\left(\sigma_{x}, \sigma_{y}, \sigma_{z}\right)$ are the Pauli matrices. The one of the consequences of the Hohenberg-Kohn theorem is that ground-state energy $E$ of $H$ in Eq. (2.1) is functional of the form

$$
E=E[\rho]=\sum_{s^{\prime}, s} \int \mathrm{d} r \rho_{s^{\prime} s}(r) v_{s s^{\prime}}(r)+\frac{1}{2} e^{2} \int \mathrm{d} r^{\prime} \mathrm{d} \boldsymbol{r} \frac{n\left(\boldsymbol{r}^{\prime}\right) n(\boldsymbol{r})}{\left|\boldsymbol{r}^{\prime}-\boldsymbol{r}\right|}+E_{\mathrm{xc}}[\rho],
$$

with contribution $E_{\mathrm{xc}}[\rho]$ which comes from exchange and correlation effects. Following the observation by Lam and Platzman, spin density matrix can be found alternatively by the approach based on Feynman's theorem. If $E(\lambda)$ is the ground-state energy of $H(\lambda)$ of the form

$$
H(\lambda)=H+\lambda \Psi^{+}\left(s^{\prime}, r\right) \Psi(s, r),
$$

then by comparing with Eq. (2.3) we get

$$
\frac{\partial E(\lambda)}{\partial \lambda}=\rho_{s^{\prime} s}(r)
$$

With another consequence of the HK-theorem that $E$ is stationary with respect to $\rho_{s s^{\prime}}(r)$ we get the Kohn-Sham set of differential equations for the members of the orthonormal set of functions $\left\{\phi_{i}\right\}$. If writing the spin density matrix as series

$$
\rho_{s s^{\prime}}(r)=\sum_{i} \phi_{i}^{*}\left(s^{\prime}, r\right) \phi_{i}(s, r) \Theta\left(\epsilon_{\mathrm{F}}-\epsilon_{i}\right) .
$$

we find that $\phi_{i}$ satisfies

$$
\sum_{s}\left[-\nabla^{2} \delta_{s^{\prime} s}+v_{\mathrm{c}}(r) \delta_{s^{\prime} s}+\mu_{s^{\prime} s}(r)+v_{s^{\prime} s}(r)\right] \phi_{i}(s, r)=\epsilon_{i} \phi_{i}(s, r)
$$

where

$$
v_{\mathrm{c}}(\boldsymbol{r})=-\sum_{n} \frac{Z_{n} e^{2}}{\left|\boldsymbol{r}-\boldsymbol{R}_{n}\right|}+e^{2} \int \mathrm{d} \boldsymbol{r}^{\prime} \frac{n\left(\boldsymbol{r}^{\prime}\right)}{\left|\boldsymbol{r}-\boldsymbol{r}^{\prime}\right|} .
$$

and

$$
\mu_{s^{\prime} s}(r)=\frac{\delta E_{\mathrm{xc}}[\rho]}{\delta \rho_{s s^{\prime}}(r)} .
$$

If looking for the spin momentum density matrix, we may proceed similarly, recalling the second quantized field operators now as

$$
\begin{aligned}
& \Psi^{+}(s, p)=\frac{1}{(2 \pi)^{3 / 2}} \int \mathrm{d} r \exp (\mathrm{i} p \cdot r) \Psi^{+}(s, r) \\
& \Psi(\mathrm{s}, p)=\frac{1}{(2 \pi)^{3 / 2}} \int \mathrm{d} r \exp (-\mathrm{i} p \cdot r) \Psi(s, r)
\end{aligned}
$$


Then instead of Eq. (2.6) we put

$$
H(\lambda)=H+\lambda \Psi^{+}(s, p) \Psi(s, p) .
$$

Then by differentiating with respect to $\lambda$ we get

$$
\frac{\partial E(\lambda)}{\partial \lambda}=\left\langle 0\left|\Psi^{+}\left(s^{\prime}, p\right) \Psi(s, p)\right| 0\right\rangle=\rho_{s s^{\prime}}(p),
$$

which is the spin momentum density matrix. Putting $\rho(p)$ in the equivalent form as in (2.4) we get

$$
\rho(p)=\frac{1}{2}[n(p) 1+m(p) \cdot \sigma]
$$

with electron momentum density $n(p)$ and spin momentum density $m(p)$. But now with the spin momentum density matrix, unlikely to the series expansion of the spin density matrix given in Eq. (2.8), the similar expansion will be only approximate. Generally, we may put this series as

$$
\rho_{s^{\prime} s}(p)=\sum_{i} \phi_{i}^{*}\left(s^{\prime}, p\right) \phi_{i}(s, p) \Theta\left(\epsilon_{\mathrm{F}}-\epsilon_{i}\right)+\Delta \rho_{s^{\prime} s}(p),
$$

where $\Delta \rho_{s^{\prime}}(p)$ means the correction functional which is formally proved [7] to be done as

$$
\Delta \rho_{s^{\prime} s}(p)=\left.\frac{\partial E_{\mathrm{xc}}[\rho](\lambda)}{\partial \lambda}\right|_{\lambda=0} .
$$

Its explicit form is a formidable task of the inhomogeneous spin-polarized fermion gas, and stands as central problem of fermiology.

\section{The spin-dependent KKR-CPA formalism for multicomponent alloys}

We consider a multicomponent alloy with basis atoms in positions $a_{k}$ $\left(k=1, \ldots, k_{\mathrm{b}}\right)$ from which the $k$-sublattices are generated by applying translational symmetry vectors $\boldsymbol{R}_{n}(n=1, \ldots, N)$ of Bravais lattice. We assume that sublattices sites can be occupied randomly by atoms $X_{k}=\left(A_{k}\right.$, or $\left.B_{k}\right)$ with concentration $c\left(A_{k}\right)$, and $c\left(B_{k}\right)=1-c\left(A_{k}\right)$, respectively. For the special case, if $c\left(A_{k}\right)=1.0$ we may think that $k$-sublattices are perfectly ordered like in crystal. The model of the effective one-electron potential we use is of the muffin-tin form, i.e., spherical inside of the non-overlapping spheres and constant outside spheres. This model proved to be very fruitful for describing electronic structure of binary alloys. In the following we focus our discussion on the matrix elements of the effective Green function calculated in Korringa-Kohn-Rostoker formalism with coherent-potential approximation. The relevant expression for the GF elements of the systems of atoms as described above is [3]

$$
\begin{aligned}
& \left\langle s^{\prime}, \boldsymbol{r}^{\prime}+\boldsymbol{a}_{k^{\prime}}+\boldsymbol{R}_{n^{\prime}}|G(E)| s, \boldsymbol{r}+\boldsymbol{a}_{k}+\boldsymbol{R}_{n}\right\rangle \\
& =-\sum_{\sigma L} J_{\sigma L}^{\left(X_{k}\right)}\left(s^{\prime} \boldsymbol{r}^{\prime}\right) Z_{\sigma L}^{\left(X_{k}\right)}(s \boldsymbol{r}) \delta_{k^{\prime} k} \delta_{n^{\prime} n} \\
& +\sum_{\sigma^{\prime} L^{\prime}, \sigma L} Z_{\sigma^{\prime} L^{\prime}}^{\left(X_{k^{\prime}}\right)}\left(s^{\prime} \boldsymbol{r}^{\prime}\right) T_{k^{\prime} \sigma^{\prime} L^{\prime}, k \sigma L}^{\left(n^{\prime}, n\right)} Z_{\sigma L}^{\left(X_{k}\right)}(s r) .
\end{aligned}
$$


In Eq. (3.1), $r^{\prime}>r$, if $r^{\prime}<r$ then $J^{\left(X_{k}\right)}$ and $Z^{\left(X_{k}\right)}$ should be transposed. $Z_{\sigma L}^{\left(X_{k}\right)}(s r)$ and $J_{\sigma L}^{\left(X_{k}\right)}(s r)$ are the regular and irregular solutions, respectively, of the Schrödinger wave equation with the spin-dependent potential of the $X_{k}$-atom

$$
\sum_{s}\left\{\left(E+\nabla^{2}\right) \delta_{s^{\prime} s}-\left[v_{0}^{\left(X_{k}\right)}(r) \delta_{s^{\prime} s}+v_{1}^{\left(X_{k}\right)}(\boldsymbol{r}) \hat{n} \cdot \sigma_{s^{\prime} s}\right]\right\} Z_{\sigma L}^{\left(X_{k}\right)}(s, r)=0 .
$$

The wave functions $Z_{\sigma L}^{\left(X_{k}\right)}(s r)$ and $J_{\sigma L}^{\left(X_{k}\right)}(s r)$ are normalized outside the muffin-tin sphere (i.e. if $r>S_{k}$ ) in the following way:

$$
\begin{aligned}
& Z_{\sigma L}^{\left(X_{k}\right)}(s r)=\sum_{\sigma^{\prime} L^{\prime}} \chi_{\sigma^{\prime}}(s) j_{l}(\sqrt{E} r) Y_{L^{\prime}}(\hat{r})\left[\tau^{\left(X_{k}\right)}\right]_{\sigma^{\prime} L^{\prime}, \sigma L}^{-1} \\
& -\mathrm{i} \sqrt{E} \chi_{\sigma}(s) h_{l}^{+}(\sqrt{E} r) Y_{L}(\hat{r}) \\
& J_{\sigma L}^{\left(X_{k}\right)}(s r)=\chi_{\sigma}(s) j_{l}(\sqrt{E} r) Y_{L}(\hat{r}) .
\end{aligned}
$$

Here $Y_{L}(\hat{r})$ is a real spherical harmonic, $L \equiv(l, m)$ are angular and magnetic quantum numbers. The quantum spin index $\sigma=(+,-)$ and spin variable $s=$ $(+,-)$ allow to treat the lattice with magnetic moments. In Eqs. (3.3a) the spin part of the wave function is $\chi_{\sigma}(s)=\delta_{\sigma s}$ and $h^{+}(x)=j_{l}(x)+i n_{l}(x)$ is spherical Hankel function resulting from combination of $j_{l}(x)$ spherical Bessel functions and $n_{l}(x)$ spherical Neumann functions. The matrix $\tau^{\left(X_{k}\right)}(E)$ is build up from the energy-shell elements of the $t$-matrix of the $X_{k}$-atom on the $k$-site $\left(X_{k}=A_{k}\right.$, or $B_{k}$ ). The elements of the matrix $\tau^{\left(X_{k}\right)}(E)$ are related to the corresponding phase shift, $\eta_{\sigma l}^{\left(X_{k}\right)}(E)$, in case of spherical symmetry given by the equation

$$
\tau_{\sigma^{\prime} L^{\prime}, \sigma L}^{\left(X_{k}\right)}(E)=-\sqrt{E} \exp \left[\mathrm{i} \eta_{\sigma l}^{\left(X_{k}\right)}(E)\right] \sin \left[\eta_{\sigma l}^{\left(X_{k}\right)}(E)\right] \delta_{\sigma^{\prime} \sigma} \delta_{L^{\prime} L}
$$

The expression for GF in Eq. (3.1) is valid for any set of the scattering atoms. If specializing to the case where $\boldsymbol{R}_{n}$-site vectors form Bravais lattice, we assume that in each Wigner-Seitz (WS) cell the configuration is one of the form $\{X\}=$ $\left\{X_{1}=\left(A_{1}, B_{1}\right), \ldots, X_{k_{\mathrm{b}}}=\left(A_{k_{\mathrm{b}}}, B_{k_{\mathrm{b}}}\right)\right\}$ with the probability $P\{X\}$ resulting from a product of concentration

$$
P\{X\}=c\left(X_{1}\right) \ldots c\left(X_{k_{\mathrm{b}}}\right) .
$$

In the spirit of CPA approximation, we put on each WS-cell the same set of effective scatterers. If we denote effective scatterer $t$-matrix for $k$-sublattice as $\tau_{\mathrm{CP}}^{(k)}(E)$, and by the tensor product matrix $\tau_{\mathrm{CP}}$

$$
\tau_{\mathrm{CP}}(E)=\tau_{\mathrm{CP}}^{(1)}(E) \otimes \ldots \otimes \tau_{\mathrm{CP}}^{\left(k_{\mathrm{b}}\right)}(E)
$$

then we may find on-energy shell $t$-matrix of the whole system from the sum over $k$-points in the first Brillouin zone (BZ)

$$
\begin{aligned}
& T^{\mathrm{CP}}(E)=\frac{1}{N} \sum_{k \in \mathrm{BZ}}\left[\tau_{\mathrm{CP}}^{-1}(E)-B(E, k)\right]^{-1}, \\
& {[B(E, k)]_{k^{\prime} L^{\prime}, k L}=\sum_{\boldsymbol{R}_{n^{\prime} n}} \exp \left(\mathrm{i} k \cdot \boldsymbol{R}_{n^{\prime} n}\right)[B(E)]_{k^{\prime} L^{\prime}, k L}^{\left(n^{\prime} n\right)} .}
\end{aligned}
$$


known as KKR-complex crystal structure functions [8], which are defined via multipole distribution of the free electron GF

$$
\begin{aligned}
\left\langle\boldsymbol{r}^{\prime}+\right. & \left.\boldsymbol{a}_{k^{\prime}}+\boldsymbol{R}_{n^{\prime}}\left|G_{0}(E)\right| \boldsymbol{r}+\boldsymbol{a}_{k}+\boldsymbol{R}_{n}\right\rangle \\
& =-\frac{1}{4 \pi} \frac{\mathrm{i} \sqrt{E}\left|\boldsymbol{r}^{\prime}+\boldsymbol{a}_{k^{\prime}}+\boldsymbol{R}_{n^{\prime}}-\left(\boldsymbol{r}+\boldsymbol{a}_{k}+\boldsymbol{R}_{n}\right)\right|}{\left|\boldsymbol{r}^{\prime}+\boldsymbol{a}_{k^{\prime}}+\boldsymbol{R}_{n^{\prime}}-\left(\boldsymbol{r}+\boldsymbol{a}_{k}+\boldsymbol{R}_{n}\right)\right|} \\
& =\sum_{L^{\prime} L} Y_{L^{\prime}}\left(\hat{r}^{\prime}\right)\left[-\mathrm{i} \sqrt{E} h_{l}^{+}\left(\sqrt{E} r_{>}\right) j_{l}\left(\sqrt{E} r_{<}\right)\right] Y_{L}(\hat{r}) \delta_{k^{\prime} k} \delta_{n^{\prime} n} \delta_{L^{\prime} L} \\
& +\sum_{L^{\prime} L} Y_{L^{\prime}}\left(\hat{r^{\prime}}\right) j_{l^{\prime}}\left(\sqrt{E} \boldsymbol{r}^{\prime}\right) B_{k^{\prime} L^{\prime}, k L}^{\left(n^{\prime} n\right)} Y_{L}(\hat{r}) j_{l}(\sqrt{E} r) .
\end{aligned}
$$

To find $\tau_{\mathrm{CP}}(E)$ matrices in Eq. (3.6) we first have to find $T^{\{X\}}$-matrices, which are on-energy shell $t$-matrices of the set of scatterers on central WS-cell,

$$
\tau_{\{X\}}(E)=\tau^{\left(X_{1}\right)}(E) \otimes \ldots \otimes \tau^{\left(X_{k_{\mathrm{b}}}\right)}(E)
$$

placed in the CPA-effective medium. The solution is resulting from the equation

$$
\left(T^{\{X\}}\right)^{-1}=\left(T^{\mathrm{CP}}\right)^{-1}-\left(\tau_{\mathrm{CP}}\right)^{-1}+\left(\tau_{X}\right)^{-1} .
$$

And finally $\tau_{\mathrm{CP}}(E)$ matrix has to be obtained by solving the CPA self-consistency condition, which we write down as

$$
\sum_{\{X\}} P\{X\} T^{\{X\}}(E)=T^{\mathrm{CP}}(E) .
$$

The number of atom configuration $\{X\}$ in the above equation, generally will be equal to $2^{k_{\mathrm{b}}}\left(k_{\mathrm{b}}-\right.$ number of sublattices). In our practice to solve Eq. (3.11) we use the iteration scheme resulting from the following equation:

$$
\begin{gathered}
\sum_{\{X\}} P\{X\}\left[\left(T_{n}^{\mathrm{CP}}\right)^{-1}-\left(\tau_{\mathrm{CP}}^{n}\right)^{-1}+\left(\tau_{\{X\}}\right)^{-1}\right]^{-1} \\
=\left[\left(T_{n}^{\mathrm{CP}}\right)^{-1}-\left(\tau_{\mathrm{CP}}^{n}\right)^{-1}+\left(\tau_{\mathrm{CP}}^{n+1}\right)^{-1}\right]^{-1} .
\end{gathered}
$$

Let us assume that at $n$-step we know $\tau_{\mathrm{CP}}^{n}$ and $T_{n}^{\mathrm{CP}}$, so $\tau_{\mathrm{CP}}^{n+1}$ can be readily found from Eq. (3.12), and subsequently used as input in the $(n+1)$-step. To find $T_{n+1}^{\mathrm{CP}}$ we must perform $k$-space integration in Eq. (3.7a). Such integration can be done very effectively with the special $k$-points techniques [9], if energy points are put on contour above real axis. Such series of $\tau_{\mathrm{CP}}^{n}$-values was proved to be absolutely convergent [3] and preserving analytical properties of resulting solution with respect to energy as complex variable. This is important because many other schemes known in the literature often fail to converge at some points and lead to more cumbersome and less reliable computational procedure, particularly in materials with complex unit cell. At this stage of computations GF is fully determined, and for the central WS-cell with $\{X\}$-configuration of atoms is given by (if $r>r^{\prime}$ then $J^{\left(X_{k}\right)}$ and $Z^{\left(X_{k}\right)}$ should be transposed)

$$
\left\langle\left\langle s^{\prime}, r^{\prime}+a_{k^{\prime}}|G(E)| s, r+a_{k}\right\rangle\right\rangle_{\{X\}}
$$




$$
\begin{aligned}
=- & \sum_{\sigma L} J_{\sigma L}^{\left(X_{k}\right)}\left(s^{\prime} r^{\prime}\right) Z_{\sigma L}^{\left(X_{k}\right)}(s r) \delta_{k^{\prime} k} \\
& +\sum_{\sigma^{\prime} L^{\prime}, \sigma L} Z_{\sigma^{\prime} L^{\prime}}^{\left(X_{k^{\prime}}\right)}\left(s^{\prime} r^{\prime}\right) T_{k^{\prime} \sigma^{\prime} L^{\prime}, k \sigma L}^{[X]}(E) Z_{\sigma L}^{\left(X_{k}\right)}(s r)
\end{aligned}
$$

and average GF over all configuration $\{X\}$ by

$$
\begin{aligned}
& \left\langle\left\langle s^{\prime}, r^{\prime}+a_{k^{\prime}}|G(E)| s, r+a_{k}\right\rangle\right\rangle \\
& \left.=\sum_{\{X\}} P\{X\}\left\langle s^{\prime}, r^{\prime}+a_{k^{\prime}}|G(E)| s, r+a_{k}\right\rangle\right\rangle_{\{X\}} .
\end{aligned}
$$

With GF as in Eq. (3.13) and Eq. (3.14) we can proceed with computations of the other relevant physical quantities. But at this point we need Fermi energy $E_{\mathrm{F}}$, and the poor determination of $E_{\mathrm{F}}$ level may affect substantially many quantities, among them the electronic charges distributions between atoms, and obscure the self-consistency solution. In our paper we use for determining $E_{\mathrm{F}}$ the Lloyd formula. From this formula the total number of states up to the given energy can be found to high accuracy. Its generalization to multi-atom alloys with the spin-dependent CPA formalism is quite straightforward and the main steps are explained in the following paragraph.

At the start we have to take trace from GF (see Eq. (3.14)) over WS-cell and spin discrete space

$$
G(E)=\sum_{s=(+,-)} \sum_{k} \int_{V_{k}} \mathrm{~d}^{3} r\left\langle\left\langle s, r+a_{k}|G(E)| s, r+a_{k}\right\rangle\right\rangle .
$$

In the integrand in Eq. (3.15) we may think that each atom is placed inside Voronoi polyhedron $V_{k}$ (instead of muffin-tin sphere) which make possible to fill up exactly WS-cell. We assume also that the magnetic structure is collinear (with the same $z$-axis of quantization on each atom). Then with the same arguments as in [5] we can prove that

$$
\begin{aligned}
G(E) & =G_{0}(E)+\frac{\mathrm{d}}{\mathrm{d} E}\left\{\frac{1}{N} \sum_{k l \in \mathrm{BZ}} \operatorname{tr} \ln \left[\tau_{\mathrm{CP}}^{-1}(E)-B(E, k)\right]^{-1}\right\} \\
+ & \frac{\mathrm{d}}{\mathrm{d} E}\left\{\sum_{\{X\}} P\{X\} \operatorname{tr} \ln \left[\phi_{\{X\}}^{-1}(E)\left(1+T^{\mathrm{CP}}\left(\tau_{\mathrm{CP}}^{-1}-\tau_{\{X\}}^{-1}\right)\right)^{-1}\right]\right\} .
\end{aligned}
$$

In Eq. (3.16) $G_{0}(E)$ is free electron contribution given by

$$
\begin{aligned}
G_{0}(E) & =\sum_{\sigma L} \sum_{k} \int_{V_{k}} \mathrm{~d}^{3} r Y_{L}(\hat{r})\left[-\mathrm{i} \sqrt{E} h_{l}^{+}(\sqrt{E} r) j_{l}(\sqrt{E} r)\right] Y_{L}(\hat{r}) \\
+ & \frac{\mathrm{d}}{\mathrm{d} E} \ln (\sqrt{E})^{l}
\end{aligned}
$$


and $\phi_{\{X\}}(E)$ is energy-dependent normalization matrix with elements

$$
\left[\phi_{\{X\}}\right]_{k^{\prime} \sigma^{\prime} L^{\prime}, k \sigma L}=\phi_{\sigma l}^{\left(X_{K}\right)}(E) \delta_{k^{\prime} k} \delta_{\sigma^{\prime} \sigma} \delta_{L^{\prime} L}
$$

where $\phi_{\sigma l}^{\left(X_{k}\right)}(E)$ is defined as

$$
Z_{\sigma l}^{\left(X_{k}\right)}(E, r)=\phi_{\sigma l}^{\left(X_{k}\right)}(E) \Psi_{\sigma l}^{\left(X_{k}\right)}(E, r)
$$

with $\Psi_{\sigma l}^{\left(X_{k}\right)}(E, r) \rightarrow r^{l}$ (if $r \rightarrow 0$ ). With the motivation that we carry our calculations in the complex energy plane, we rewrite $G(E)$ in the form which was proved to be very convenient for numerical implementation. For this purpose we write the logarithmic derivative of the $X_{k}$-atom radial wave functions, as

$$
D_{\sigma l}^{\left(X_{k}\right)}(E)=\left.S_{k}^{2} \frac{\partial}{\partial r} \ln Z_{\sigma l}^{\left(X_{k}\right)}(E, r)\right|_{r=S_{k}}
$$

and relate the corresponding $\tau$-matrices in Eq. (3.4) with

$$
\begin{aligned}
& \left(\tau_{\sigma l}^{\left(X_{k}\right)}(E)\right)^{-1}=\frac{1}{j_{l}\left(\sqrt{E} S_{k}\right)} \frac{1}{D_{\sigma l}^{\left(X_{k}\right)}(E)-D_{k l}^{(j)}(E)} \frac{1}{j_{l}\left(\sqrt{E} S_{k}\right)} \\
& \quad+\mathrm{i} \sqrt{E} \frac{h_{l}^{+}\left(\sqrt{E} S_{k}\right)}{j_{l}\left(\sqrt{E} S_{k}\right)}
\end{aligned}
$$

where

$$
D_{k l}^{(j)}(E)=\left.S_{k}^{2} \frac{\partial}{\partial r} \ln j_{l}(\sqrt{E} r)\right|_{r=S_{k}} .
$$

We also require the angular-momentum representation of free-electron GF with position vectors on muffin-tin spheres

where

$$
\begin{gathered}
{\left[G_{0}(E, k)\right]_{k^{\prime} \sigma^{\prime} L^{\prime}, k \sigma L}=\left[\frac{1}{D^{(h)}-D^{(j)}}\right]_{k l} \delta_{k^{\prime} k} \delta_{\sigma^{\prime} \sigma} \delta_{L^{\prime} L}} \\
+j_{l^{\prime}}\left(\sqrt{E} S_{k^{\prime}}\right)[B(E, k)]_{k^{\prime} L^{\prime}, k L} j_{l}\left(\sqrt{E} S_{k}\right) \delta_{\sigma^{\prime} \sigma}
\end{gathered}
$$

$$
D_{k l}^{(h)}(E)=\left.S_{k}^{2} \frac{\partial}{\partial r} \ln h_{l}^{+}(\sqrt{E} r)\right|_{r=S_{k}} .
$$

Eliminating the $\tau$ and $B$-matrices in Eq. (3.15) in favor of $G_{0}(E, k)$ and

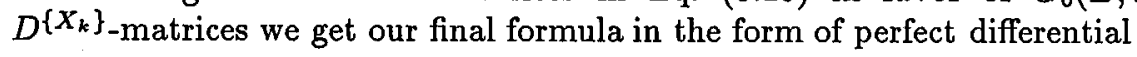

$$
\begin{gathered}
G(E)=-\frac{\mathrm{d}}{\mathrm{d} E} \frac{1}{N} \sum_{k \in \mathrm{BZ}} \operatorname{tr} \ln \left[G_{0}^{-1}(E, k)+D_{j}-D_{\mathrm{CP}}\right]^{-1} \\
-\frac{\mathrm{d}}{\mathrm{d} E} \sum_{\{X\}} P\{X\} \operatorname{tr} \ln \left[\Psi_{\{X\}}^{-1} G^{\{X\}}\left(G^{\mathrm{CP}}\right)^{-1}\right] .
\end{gathered}
$$

In Eq. (3.25) matrices are indexed with $(k \sigma L)$ and are defined as

$$
\begin{aligned}
& {\left[\Psi_{\{X\}}\right]_{k^{\prime} \sigma^{\prime} L^{\prime}, k \sigma L}=\Psi_{\sigma l}^{\left(X_{k}\right)}\left(E, S_{k}\right) \delta_{k^{\prime} k} \delta_{\sigma^{\prime} \sigma} \delta_{L^{\prime} L},} \\
& G^{\mathrm{CP}}=\frac{1}{N} \sum_{k \in \mathrm{BZ}}\left[G_{0}^{-1}(E, k)+D_{j}-D_{\mathrm{CP}}\right]^{-1},
\end{aligned}
$$




$$
\begin{aligned}
& {\left[D_{j}(E)\right]_{k^{\prime} \sigma^{\prime} L^{\prime}, k \sigma L}=D_{k l}^{j}(E) \delta_{k^{\prime} k} \delta_{\sigma^{\prime} \sigma} \delta_{L^{\prime} L},} \\
& {\left[D_{\mathrm{CP}}(E)\right]_{k^{\prime} \sigma^{\prime} L^{\prime}, k \sigma L}=D_{k l}^{\mathrm{CP}}(E) \delta_{k^{\prime} k} \delta_{\sigma^{\prime} \sigma} \delta_{L^{\prime} L},} \\
& \left(G^{\{X\}}\right)^{-1}=\left(G^{\mathrm{CP}}\right)^{-1}+D_{\mathrm{CP}}^{-1}-D_{\{X\}}^{-1} .
\end{aligned}
$$

It is interesting to note that resulting from Eq. (3.25) the total number of states

$$
N(E)=-\frac{1}{\pi} \operatorname{Im} \int_{-\infty}^{E_{\mathrm{F}}} \mathrm{d} E G(E)
$$

gives all states below energy $E$ including bound states due to core electrons, which are present in the second term of Eq. (3.25). This means that prior to any density of states (DOS) computation we are able to find very accurately $E_{\mathrm{F}}$ satisfying condition $N\left(E_{\mathrm{F}}\right)=Z$ (with $Z$ equal to the number of electrons in WS-cell). For the collinear magnetic structure formula Eq. (3.15) and subsequently Eq. (3.25) can be split into two for each spin direction $(\sigma=+,-)$ separately, resulting into spin resolved DOS-functions

$$
n_{\sigma}(E)=\frac{\partial}{\partial E} N_{\sigma}(E),
$$

and the magnetic moment $\mu_{\mathrm{WS}}$ of WS-cell

$$
\mu_{\mathrm{WS}}=N_{+}\left(E_{\mathrm{F}}\right)-N_{-}\left(E_{\mathrm{F}}\right) .
$$

The computation of the spin-dependent charge density on the $k$-site with $X_{k}$-atom is done using

$$
n_{\sigma}^{\left(X_{k}\right)}(\boldsymbol{r})=-\frac{1}{\pi} \operatorname{Im} \int_{-\infty}^{E_{F}} \mathrm{~d} E\left\langle\left\langle\sigma, r+\boldsymbol{a}_{k}|G(E)| \sigma, r+\boldsymbol{a}_{k}\right\rangle\right\rangle_{\left\{X_{k}\right\}} .
$$

At this stage of computations we have collected all charges distribution in a system and we are ready to construct new effective electron potential. This is achieved using LSD approximation for computing exchange-correlation part of the potential and applying the Madelung procedure to solve Poisson equation with the crystal boundary conditions. The iteration of this procedure leads to the fully charge and spin self-consistent KKR-CPA solution. We emphasize that all steps of the described procedure are formally proved to be correct, and already fully implemented into our computer code.

\section{Spin density in momentum space}

Our interest here is mostly in the average electron spin momentum density, which is related to the measured magnetic Compton scattering profiles possibly in multi-atom random alloys. Assuming that all computations were successfully completed in the real space, and the GF constructed, we may find all relevant matrix elements in momentum space via Fourier transformation, i.e.

$$
\left\langle s^{\prime}, p^{\prime}|G(E)| s, p\right\rangle
$$




$$
=\frac{1}{(2 \pi)^{3}} \int \mathrm{d}^{3} r^{\prime} \mathrm{d}^{3} r \exp \left(\mathrm{i} p^{\prime} \cdot r^{\prime}\right)\left\langle s^{\prime}, r^{\prime}|G(E)| s, r\right\rangle \exp (-\mathrm{i} p \cdot r) .
$$

Assuming that we found $T^{\mathrm{CP}}$ satisfying Eq. (3.11) we may proceed with putting GF into momentum space at least, by attempting Fourier transform directly on Eq. (3.14). But going along this way the calculations soon become complicated, as we will need the integration also over the interstitial region, where GF function is defined implicitly. Instead, it is easier to start with the Dyson equation, following the method developed by Mijnarends and Bansil [10],

$$
G=G_{0}+G_{0} V G
$$

or equivalently

$$
G=G_{0}+G_{0} T G_{0},
$$

where $T$-operator is defined in the standard way

$$
T=V+V G_{0} T
$$

with $V$ in the form as in Eq. (3.2). The free-electron GF is well known

$$
\left\langle s^{\prime}, p^{\prime}\left|G_{0}(E)\right| s, p\right\rangle=\frac{\delta\left(p^{\prime}-p\right)}{E-p^{2}} \delta_{s^{\prime}} s .
$$

In paper [11] Bansil et al. show that expression for $G^{\mathrm{CP}}$ can be obtained directly by placing a CP-atom at every lattice-site in the alloy (in our case we put CP-cluster of atoms with $\tau_{\mathrm{CP}}$-matrix) and look for $G^{\mathrm{CP}}$ which can be found from

$$
\begin{aligned}
& \left\langle s^{\prime}, p^{\prime}\left|G^{\mathrm{CP}}(E)\right| s, p\right\rangle \\
& \quad=\frac{1}{E-p^{2}} \delta\left(p^{\prime}-p\right) \delta_{s^{\prime}}+\frac{1}{E-p^{\prime 2}}\left\langle s^{\prime}, p^{\prime}\left|T^{\mathrm{CP}}(E)\right| s, p\right\rangle \frac{1}{E-p^{2}} .
\end{aligned}
$$

Including electron-spin and allowing for few nonequivalent atoms in WS-cell, but with restrictions that we have alloying only on one of the sublattice with $A$, or $B$ atoms $T^{\mathrm{CP}}$ has the following form:

$$
\begin{aligned}
& \left\langle s^{\prime}, p^{\prime}\left|T^{\mathrm{CP}}(E)\right| s, p\right\rangle=\frac{(4 \pi)^{2}}{\Omega_{\mathrm{WS}}} \sum_{\sigma^{\prime} L^{\prime}, \sigma L} \chi_{\sigma^{\prime}}\left(s^{\prime}\right) Y_{L^{\prime}}\left(\hat{p}^{\prime}\right)\left\{\left[t_{\mathrm{CP}}\left(p^{\prime}, p\right)\right.\right. \\
& \left.-t_{\mathrm{CP}}\left(p^{\prime}, \kappa\right) \tau_{\mathrm{CP}}^{-1}(\kappa) t_{\mathrm{CP}}(\kappa, p)\right]_{\sigma^{\prime} L^{\prime}, \sigma L}+\left[t_{\mathrm{CP}}\left(p^{\prime}, \kappa\right) \tau_{\mathrm{CP}}^{-1}(\kappa)\left(\tau_{\mathrm{CP}}^{-1}(\kappa)-B(k, E)\right)^{-1}\right. \\
& \left.\left.\times \tau_{\mathrm{CP}}^{-1}(\kappa) t_{\mathrm{CP}}(\kappa, p)\right]_{\sigma^{\prime} L^{\prime}, \sigma L}\right\} \chi_{\sigma}(s) Y_{L}(\hat{p}) .
\end{aligned}
$$

In Eq. (4.7) matrix $\tau_{\mathrm{CP}}(\kappa)(\kappa=\sqrt{E})$ is on-shell energy standard CPA solution, as described in Eq. (3.12). Then the off-shell elements of $t_{\mathrm{CP}}\left(p^{\prime}, p\right)$ are given by (see Bansil et al. [11], Eq. (2.14))

$$
\begin{aligned}
& t_{\mathrm{CP}}\left(p^{\prime}, p\right)=\left\langle t\left(p^{\prime}, p\right)\right\rangle-\left[t_{A}\left(p^{\prime}, \kappa\right)-t_{\mathrm{CP}}\left(p^{\prime}, \kappa\right)\right] \\
& \quad \times \frac{1}{N} \sum_{k \in \mathrm{BZ}}\left[B^{-1}(k, E)-\tau_{\mathrm{CP}}(\kappa)\right]^{-1}\left[t_{B}(\kappa p)-t_{\mathrm{CP}}(p, \kappa)\right] .
\end{aligned}
$$


The advantages of using the above form of GF in Eq. (4.6) for the momentum density calculations

$$
n_{\sigma}(p)=-\frac{1}{\pi} \operatorname{Im} \int_{-\infty}^{E_{\mathrm{F}}} \mathrm{d} E\left\langle\sigma, p\left|G^{\mathrm{CP}}(E)\right| \sigma, p\right\rangle
$$

comes out from the fact that it is strictly preserving the physically essential Herglotz properties. Thus the integral interval in Eq. (4.9) can be moved to upper part of complex energy plane in the similar way as in the case of the spin densities in coordinate space. But the price for use of the $T$-matrix based algorithm for computing momentum densities is that we have to know the off-shell atomic scattering elements $t\left(p^{\prime}, p\right)$ (also, for complex value of energy $E$ ). As this task is nontrivial, we will describe the way this problem is solved and applied in our computation practice. First we recall the assumption that our potential is already in spherical form with muffin-tin shape. With all self-consistency steps done, we have on each atom $X_{k}$, this potential $v_{\sigma}^{\left(X_{k}\right)}(r)$ for both spin-direction $\sigma$. Let us look now first on the following integral equation:

$$
S_{\sigma l}^{\left(X_{k}\right)}(r, p)=j_{l}(p, r)+\int \mathrm{d} r^{\prime} r^{\prime 2} g_{l}\left(r, r^{\prime}\right) v_{\sigma}^{\left(X_{k}\right)}\left(r^{\prime}\right) S_{\sigma l}^{\left(X_{k}\right)}\left(r^{\prime}\right)
$$

where

$$
g_{l}\left(r, r^{\prime}\right)=-\mathrm{i} \kappa h_{l}\left(\kappa r_{>}\right) j_{l}\left(\kappa r_{<}\right)
$$

is the radial part of the free-electron GF. With the definition of the $t$-operator we may write

$$
\begin{aligned}
& t_{\sigma l}^{\left(X_{k}\right)}\left(r^{\prime}, r\right) \\
& \quad=v_{\sigma}^{\left(X_{k}\right)}\left(r^{\prime}\right) \delta\left(r^{\prime}, r\right)+\int \mathrm{d} r^{\prime 2} r^{\prime \prime 2} t_{\sigma l}^{\left(X_{k}\right)}\left(r^{r^{\prime}}, r^{\prime \prime}\right) g_{l}\left(r^{\prime \prime}, r^{\prime}\right) v_{\sigma}^{\left(X_{k}\right)}(r),
\end{aligned}
$$

which shows if comparing to Eq. (4.10) that

$$
t_{\sigma l}^{\left(X_{k}\right)}(r, p)=v_{\sigma}^{\left(X_{k}\right)}(r) S_{\sigma l}^{\left(X_{k}\right)}(r, p)
$$

and finally

$$
t_{\sigma l}^{\left(X_{k}\right)}\left(p^{\prime}, p\right)=\int \mathrm{d} r r^{2} j_{l}\left(p^{\prime}, r\right) v_{\sigma}^{\left(X_{k}\right)}(r) S_{\sigma l}^{\left(X_{k}\right)}(p, r) .
$$

The formulas in Eqs. (4.13) and (4.14) allow us to find any off-shell element of the $t$-matrix as soon as we find the function $S_{\sigma l}^{\left(X_{k}\right)}(r, p)$. If putting $p=\kappa$ (on energy-shell) $S_{\sigma l}^{\left(X_{k}\right)}(r, \kappa)$ becomes the usual wave function with asymptotic form

$$
R_{\sigma l}^{\left(X_{k}\right)}(r)=S_{\sigma l}^{\left(X_{k}\right)}(r, p=\kappa)=j_{l}(\kappa r)-\mathrm{i} \kappa h_{l}(\kappa r) \tau_{\sigma l}^{\left(X_{k}\right)}(\kappa) .
$$

For off-shell momentum $p \neq \kappa$ we found convenient to introduce another function $O_{\sigma l}^{\left(X_{k}\right)}(r, p)$ defined as

$$
S_{\sigma l}^{\left(X_{k}\right)}(r, p)=\left(E-p^{2}\right) O_{\sigma l}^{\left(X_{k}\right)}(r, p)+w R_{\sigma l}^{\left(X_{K}\right)}(r) .
$$

In Eq. (4.16) the constant value $w$ is given later in Eq. (4.18). From the fact that $S_{\sigma l}(r, p)$ satisfy integral equation $(4.10)$ results

$$
\left[\frac{\mathrm{d}^{2}}{\mathrm{~d} r^{2}}+E-v_{\sigma}^{\left(X_{k}\right)}(r)-\frac{l(l+1)}{r^{2}}\right] r O_{\sigma l}^{\left(X_{k}\right)}(r, p)=r j_{l}(p, r)
$$




$$
\left[\frac{\mathrm{d}^{2}}{\mathrm{~d} r^{2}}+E-v_{\sigma}^{\left(X_{k}\right)}(r)-\frac{l(l+1)}{r^{2}}\right] r R_{\sigma l}^{\left(X_{k}\right)}(r)=0 .
$$

To get function $S_{\sigma l}^{\left(X_{k}\right)}(r, p)$ with asymptotic form like in Eq. (4.15) we must assume for the constant $w$ in Eq. (4.16) the following value:

$$
\begin{aligned}
w= & W\left\{j_{l}(p r)-\left(E-p^{2}\right) O_{\sigma l}^{\left(X_{k}\right)}(r, p),-\mathrm{i} \kappa h_{l}(\kappa r)\right\}_{r=S_{k}} \\
& / W\left\{R_{\sigma l}^{\left(X_{k}\right)}(r),-\mathrm{i} \kappa h_{l}(\kappa r)\right\}_{r=S_{k}},
\end{aligned}
$$

where $W\{f, g\}$ means Wronskian of two functions. The numerical procedures used to solve differential equations in (4.17) are well established (e.g. Numerov method) and can be applied to get $R_{\sigma l}^{\left(X_{k}\right)}(r), O_{\sigma l}^{\left(X_{k}\right)}(r, p)$ and subsequently $S_{\sigma l}^{\left(X_{k}\right)}(r, p)$ in (4.10). Moreover, the energy value $E$ does not complicate numerical procedure if it becomes complex. The radial integrals in Eq. (4.14) are all one-dimensional and are found numerically very fast.

\section{Illustrative examples}

Charge and spin self-consistent KKR-CPA calculations were carried out for the number of the transition metals and their alloys, but here we first describe the results of our computing for $\mathrm{Fe}$ and $\mathrm{Fe}_{1-x} \mathrm{Si}_{x}(x=0.058)$ alloys, with cubic (bcc) structure. As already described in Sec. 3 calculations yield electron spin densities, which are mostly due to electrons with energy close to Fermi energy $E_{\mathrm{F}}$. These results for radial spin densities are presented in Fig. 1. We observe here that adding of $\mathrm{Si}$ in place of Fe changes only a little the spin densities on both atoms. It reflects the fact that at low concentrations of $\mathrm{Si}$ the spatial disturbance is of the short range, and on average majority of $\mathrm{Fe}$ atoms are unaffected. To contrast this observation supported in Fig. 1, we present in Fig. 2 all three momentum densities (spin up, spin down and their difference) along the $\langle 1,0,0\rangle$ direction with starting point at origin. We find by inspecting Fig. 2 that adding of Si $(x=0.058)$ makes, at first, all breaks on momentum curves more diffusive. Secondly, for the spin-up momentum density adding of $\mathrm{Si}$ induces a structure with a sharp peak around momentum 0.8 a.u. This fact reflects the strong changes of the Fermi surface for the spin-up electrons. But unfortunately, when performing the projection in Eq. (5.1)

$$
J_{\sigma}(p)=\int \mathrm{d} p^{\prime} n_{\sigma}\left(p^{\prime}\right) \delta\left(p-\hat{n} \cdot p^{\prime}\right)
$$

of the momentum density $n_{\sigma}(\boldsymbol{p})$ on the chosen direction $\hat{n}$ and getting the Compton profile $J_{\sigma}(p)$ most of that structure is lost. The last remark was drawn out by observing results shown in Fig. 3. Here, we made plots of magnetic Compton profiles $J_{\text {mag }}(p)=J_{+}(p)-J_{-}(p)$ for the three directions $\langle 1,0,0\rangle,\langle 1,1,0\rangle$ and $\langle 1,1,1\rangle$. These profiles reveal remarkable anisotropy, with the most pronounced differences on alloying for the $\langle 1,1,1\rangle$ direction. The similar calculations by full potential linearized augmented plane wave (FLAPW) method (but only for Fe), together with comparison with experimental high-resolution ( 0.12 a.u.) magnetic Compton profile of $\mathrm{Fe}_{1-x} \mathrm{Si}_{x}(x=0.058)$ were presented in [12]. The authors 

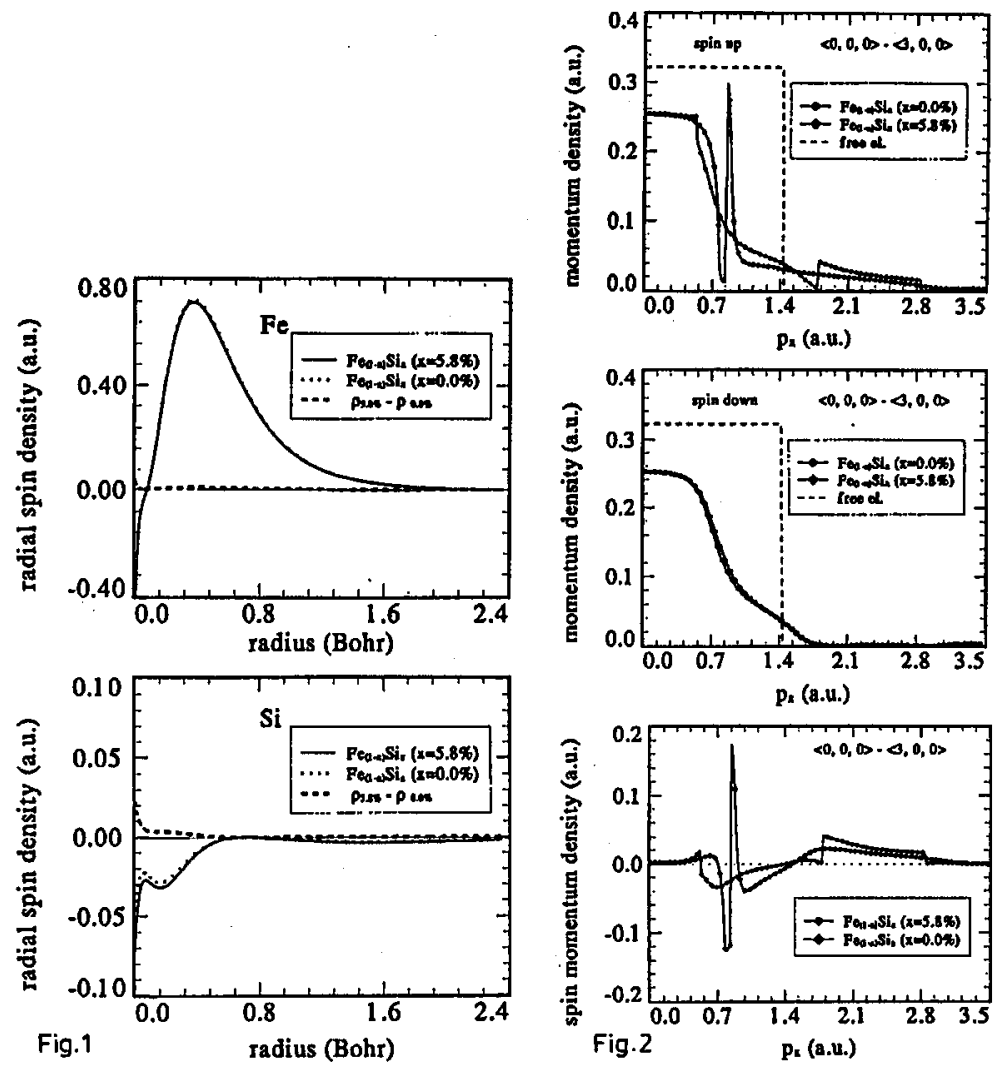

Fig. 1. Radial spin densities of $\mathrm{Fe}$ and $\mathrm{Si}$ atoms in $\mathrm{Fe}_{1.0} \mathrm{Si}_{0.0}$ (Si impurity in $\mathrm{Fe}$ ) and $\mathrm{Fe}_{0.942} \mathrm{Si}_{0.058}$ alloys.

Fig. 2 Momentum densities (by KKR-CPA method) in $\mathrm{Fe}_{1.0} \mathrm{Si}_{0.0}$ and $\mathrm{Fe}_{0.942} \mathrm{Si}_{0.058}$ along the line in momentum space from the point $\langle 0,0,0\rangle$ to the point $\langle 3,0,0\rangle$, with spin-up (upper plot), spin-down (middle plot) and their differences (bottom plot).

concluded that the results shows fairly good agreement between the theory and the experiment, except the shoulder around 1.0 a.u. In order to give more insight on the structure seen on magnetic Compton profiles in Fig. 3, we give in Fig. 4a all three maps of momentum density in $\mathrm{Fe}$ in the plane normal to $\langle 0,0,1\rangle$ ( $x y$-plane). When looking at plots in Fig. 4a we find the differences in the structure for spin-up and spin-down maps, as originating from differences in the shape of the corresponding Fermi surfaces. Then these differences are transferred onto spin momentum density, with a valley of negative values and high pile of positive values near $\left(p_{x}=0.5\right.$, $p_{y}=0.5$ ). Such shape of the spin momentum field corresponds well to the dip on the magnetic Compton profiles along $\langle 1,0,0\rangle$ direction.

As the next interesting example we take a case from our KKR-CPA calculations [5] for $\mathrm{Co}_{2-x} \mathrm{Fe}_{x} \mathrm{MnSi}$. These materials in the whole range of concentration $(x=0.0$ to 2.0 ) belongs to the interesting class of halfmetallic ferromagnets, with 


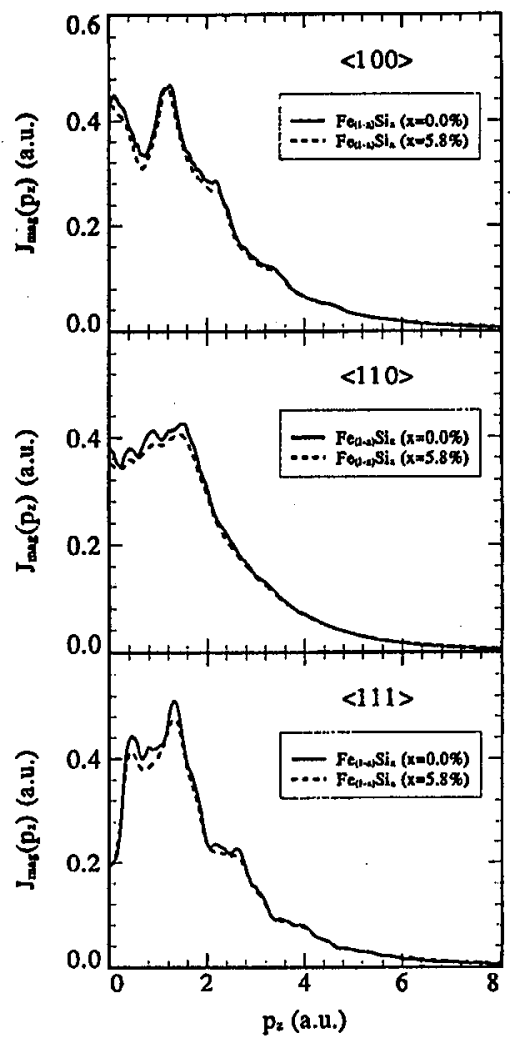

Fig. 3. Magnetic Compton profiles of $\mathrm{Fe}_{1.0} \mathrm{Si}_{0.0}$ and $\mathrm{Fe}_{0.942} \mathrm{Si}_{0.058}$ along the $\langle 1,0,0\rangle,\langle 1,1,0\rangle$ and $\langle 1,1,1\rangle$ directions.

the gap for the spin-down electrons, as it can be seen in Fig. 5, where we plot total densities of states for both directions of spin in $\mathrm{Co}_{2} \mathrm{MnSi}$. The similar plots, as in Fig. $4 \mathrm{a}$ for $\mathrm{Fe}$ were made also for the compound $\mathrm{Co}_{2} \mathrm{MnSi}$, and they are shown in Fig. $4 \mathrm{~b}$. When inspecting Fig. $4 \mathrm{~b}$, it is most interesting to note that the shape of surface for the spin-down momentum is smooth, without any breaks. This behavior only reflects the lack of the Fermi surfaces in semiconducting state. The deepest gradients are at positions of crossing $x y$-plane with Brillouin zone boundary. On the spin-up momentum density plot we see breaks in the form of the flat vertical walls, which are effects of emptying states not surrounded by Fermi surface. This is an origin of the structure seen in the plot for the spin momentum density in bottom picture in Fig. 4b. Unfortunately, much of this structure will be obscured by performing projection on the scattering vector, and making comparison with experiment less conclusive. 

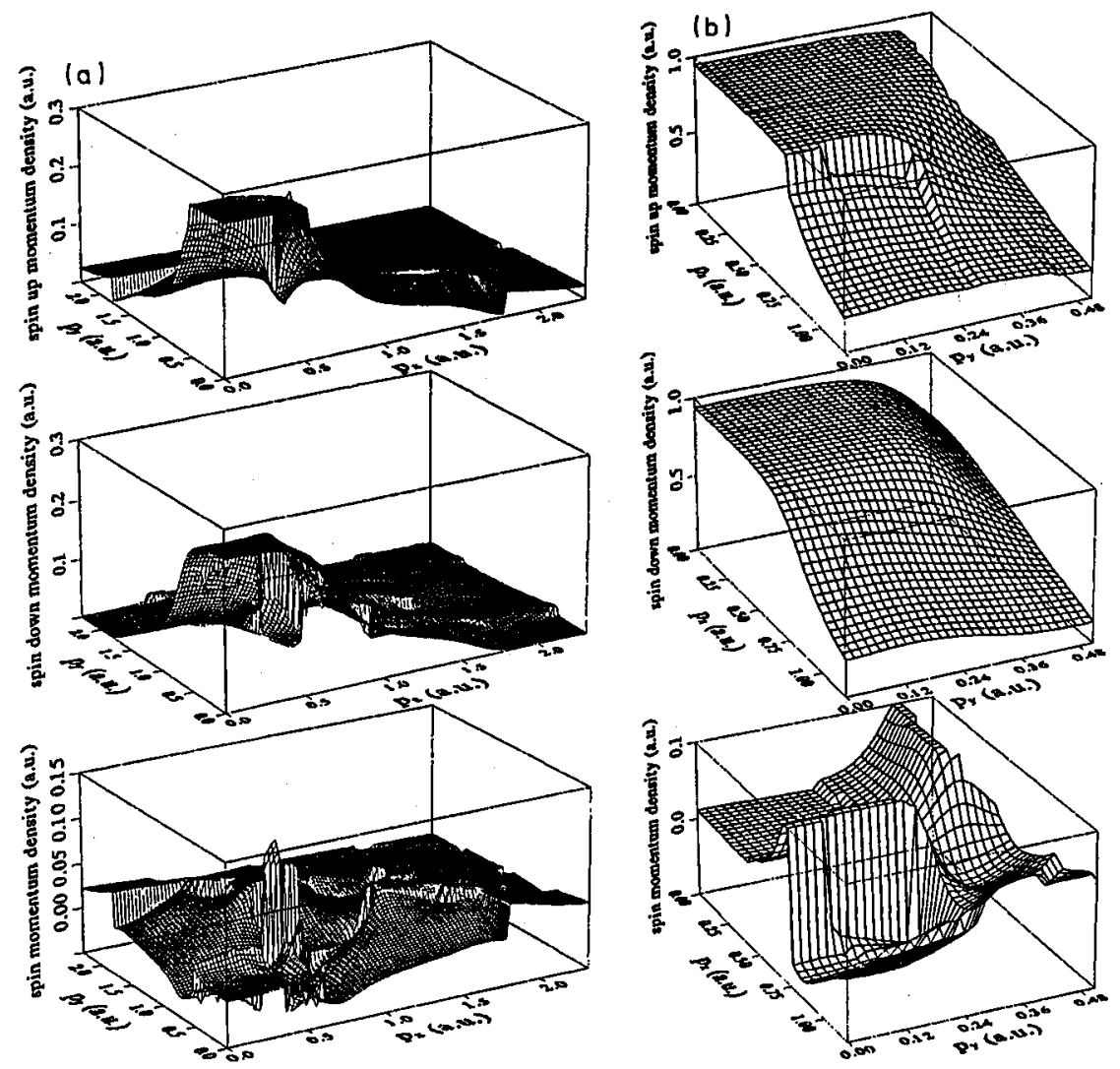

Fig. 4. Momentum densities in the momentum $x y$-plane for the electrons with spin-up, spin-down and their difference in: $\mathrm{Fe}(\mathrm{a})$ and $\mathrm{Co}_{2} \mathrm{MnSi}(\mathrm{b})$.

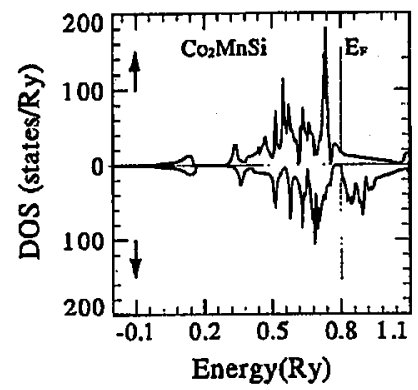

Fig. 5. Total densities of states in $\mathrm{Co}_{2} \mathrm{MnSi}$ for the electrons with spin-up and spin-down polarization. 


\section{Acknowledgments}

This work was sponsored by the Committee for Scientific Research through the grant no. 2 P302 10307.

\section{References}

[1] B.L. Gyorffy, A.J. Pindor, J. Staunton, G.M. Stocks, H. Winter, J. Phys. F, Met. Phys, 15, 1337 (1985).

[2] Applications of Multiple Scattering Theory to Materials Science, Eds. W.H. Butler, P.H. Dederichs, A. Gonis, R.L. Weawer, Vol. 253, Materials Research Society Symposium Proceedings, 1991.

[3] L. Schwartz, A. Bansil, Phys. Rev. B 21, 4322 (1980); T. Kaplan, P.L. Leath, L.J. Gray, H.W. Diehl, Phys. Rev. B 21, 4230 (1980); R. Mills, L.J. Gray, T. Kaplan, Phys. Rev. B 27, 3252 (1982).

[4] V.L. Moruzzi, J.P. Janak, A.R. Williams, Calculated Electronic Properties of Metals, Pergamon Press, Oxford 1978.

[5] S. Kaprzyk, A. Bansil, Phys. Rev. B 42, 7358 (1990).

[6] L. Lam, P.M. Platzman, Phys. Rev. B 9, 5122 (1974).

[7] G.E.W. Bauer, Phys. Rev. B 27, 5912 (1983).

[8] B. Segal, Phys. Rev. 105, 108 (1957).

[9] D.J. Chadi, M.L. Cohen, Phys. Rev. B 8, 5747 (1973); H.J. Monkhorst, J.D. Pack, Phys. Rev. B 13, 5188 (1976).

[10] P.E. Mijnarends, A. Bansil, Phys. Rev. B 13, 2381 (1976).

[11] A. Bansil, R.S. Rao, P.E. Mijnarends, L. Schwartz, Phys. Rev. B 23, 3608 (1981).

[12] Y. Sakurai, Y. Tanaka, T. Ohata, Y. Watanbe, S. Nanao, Y. Ushigami, T. Iwazumi, H. Kawata, N. Shiotani, J. Phys., Condens. Matter 6, 9469 (1994). 